\title{
An Improved Algorithm to Predict the Pose- dependent Cutting Stability in Robot Milling
}

\section{Long Wu}

Tianjin University

Guofeng Wang ( $\nabla$ gfwangmail@tju.edu.cn )

Tianjin University

Haitao Liu

Tianjin University

\section{Tian Huang}

Tianjin University and The University of Warwick

\section{Research Article}

Keywords: Robot dynamics, milling, Chatter stability analysis, Improved full-discretization method

Posted Date: August 6th, 2021

DOl: https://doi.org/10.21203/rs.3.rs-772052/v1

License: (c) (i) This work is licensed under a Creative Commons Attribution 4.0 International License. Read Full License 


\title{
An Improved Algorithm to Predict the Pose-dependent Cutting Stability in Robot Milling
}

\author{
Long Wu ${ }^{\mathrm{a}}$, Guofeng Wang ${ }^{\mathrm{a},}$ *, Haitao Liu ${ }^{\mathrm{a}}$, Tian Huang ${ }^{\mathrm{a}, \mathrm{b}}$ \\ ${ }^{a}$ Key laboratory of Modern Mechanisms and Equipment Design of The State Ministry of Education, Tianjin University, Tianjin 300072, China \\ ${ }^{\mathrm{b}}$ School of Engineering, The University of Warwick, Coventry CV4 7AL, UK
}

\begin{abstract}
Chattering is one of the most important factors affecting productivity of robot machining. This paper investigates the pose-dependent cutting stability of a 5-DOF hybrid robot. By merging the complete robot structural dynamics with the cutting force at TCP, an effective approach for stability analysis of the robot milling process is proposed using the full-discretization technique. The proposed method enables the computational efficiency to be significantly improved because the system transition matrix can be simply generated using a sparse matrix multiplication. Both simulation and experimental results on a full size prototype machine show that the stability lobes are highly pose-dependent and primarily dominated by the lower-order structural modes.
\end{abstract}

Keywords: Robot dynamics; milling; Chatter stability analysis; Improved full-discretization method

\section{Introduction}

Thanks to many advantages such as lower cost, acceptable accuracy, flexibility and reconfigurability, industrial robots have become popular for the in-situ large parts manufacturing, light machining, milling and drilling for example [1]. However, due to the pose-dependent static and dynamic behaviors [3, 4], it is an essential requirement to develop an effective algorithm that enables the cutting stability to be rapidly and accurately predicted for the optimization of cutting parameters and the selection of suitable configurations.

Cutting stability analysis in robot machining has been intensively investigated in recent years [5-11]. Similar to conventional machine tools, two types of chatters can be encountered in practices, i.e. the regenerative chatter and the mode coupling chatter. The former results from the modulation of the chip thickness caused by the repeated cutting on the same surface [4], and the latter occurs if the structural modes are closely matched in the principal axes [5]. Pan et al. [6] seems to be the first to investigate the chatter mechanism in robot milling, and concluded that the cutting instability is mainly caused by the lower order mode coupling effect. Cordes et al. [7] carried out an experimental investigation on an articulated robot in various cutting conditions. The results show that the regenerative chatter is primarily dominated by the local tool-spindle modes at high milling speed, while the mode coupling chatter is mainly dominated by the lower-order structural modes at low milling speed.

Different from conventional machine tools, the lower-order dynamics of a robotic system is highly pose-dependent [8, 9]. This feature requires the cutting stability over entire workspace to be predicted efficiently and accurately. In the past decades, various algorithms for stability prediction have been developed [10-20], and the most representative ones are the semi-discretization method (SDM) [15-17] and full-discretization method (FDM) [18-20]. The former formulates the response function of the robot milling dynamics as the delayed differential equations (DDEs) using direct integration scheme. By means of linear approximation of the delay items and zero order approximation of time-period directional matrix, the DDEs are transformed into ordinary differential equations (ODEs). After the construction of the transition matrix over one time period, the stability can then be determined by the Floquet theory. Based on the SDM, Cordes et al. [7] predicted stable boundaries affected by lower-order structural modes of the robot milling in low radial immersion condition with high accuracy. But the computational efficiency is low due to the repeated calculation of the involved matrix exponentials. The FDM simultaneously approximates the state, delay and time-periodic items in response function with linear interpolation. This treatment dramatically reduces the computational time with nearly the same accuracy compared with the SDM [18-20]. However, there still exist a huge number of high-dimensional matrices multiplications in the construction of transition matrix by FDM. The stability analysis of the robot milling with consideration of multiple order modes over entire workspace is still time-consuming.

Taking a novel 5 DOF hybrid robot named TriMule as an example, an improved algorithm is proposed to realize rapid and accurate prediction of pose-dependent stability over entire workspace. Combined with dynamic model built in

\footnotetext{
${ }^{*}$ Corresponding author.

E-mail addresses: gfwangmail@tju.edu.cn (G. Wang)
} 
Section 2, a dynamic milling process model is presented in Section 3 to illustrate the pose-dependent characteristic of hybrid robot. In Section 4, the improved algorithm considering both efficiency and accuracy is depicted by introducing sparse matrix multiplication in the course of constructing transition matrix. Experimental verifications are conducted in Section 5 to show the effectiveness of the proposed method and some useful conclusions are given in the last section.

\section{Dynamic modeling}

Fig. 1 shows a 3D view of the TriMule robot under consideration. The robot essentially consists of a 1T2R (T-Translation, R-Rotation) spatial parallel mechanism for positioning and an $\mathrm{A} / \mathrm{C}$ wrist for orientating. A spindle is attached to the rotary part of the A-axis, on which a tool-tool holder system is installed. In order to formulate dynamic equations of both robot and too-tool holder in the same coordinate frame, a body-fixed frame $\mathcal{K}_{C}$ is attached to the tool center point (TCP) $C$ with the $w$ axis coincident with the tool axis, and the $u$ axis parallel to the A-axis as clearly depicted in Fig.1. Hereafter, we assume that all vectors and matrices are expressed in $\mathcal{K}_{C}$ unless indicated otherwise. For the detailed description of the robot, please refer to [21, 22].

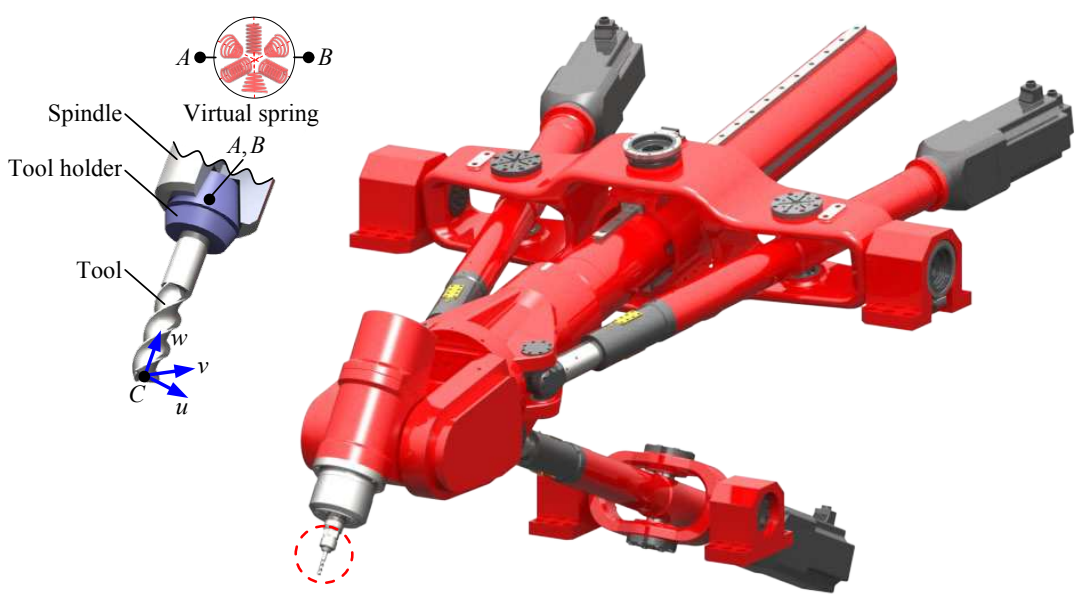

Fig.1. 3D view of the TriMule robot

In order to investigate the pose dependent cutting stability of the robot, it is necessary to develop a complete model of the system by considering: (1) dynamics of the robot itself, (2) dynamics of the tool-tool holder, and (3) dynamics of the interface between the spindle and tool holder.

We model first dynamics of the robot using the semi-analytical approach proposed in Ref. [23] such that the full set of lower order modes affecting dynamic compliances at TCP can be predicted effectively and accurately using only fourteen generalized coordinates. Consequently, the governing equations of the robot can be formulated by

$$
\left[\begin{array}{cc}
\boldsymbol{M}_{\xi_{A} \xi_{A}} & \boldsymbol{M}_{\xi_{A} \rho} \\
\boldsymbol{M}_{\xi_{A} \rho}^{\mathrm{T}} & \boldsymbol{M}_{\rho \rho}
\end{array}\right]\left(\begin{array}{c}
\ddot{\boldsymbol{\xi}}_{A} \\
\ddot{\boldsymbol{\rho}}
\end{array}\right)+\left[\begin{array}{cc}
\boldsymbol{K}_{\xi_{A} \xi_{A}} & \boldsymbol{K}_{\xi_{A} \rho} \\
\boldsymbol{K}_{\xi_{A} \rho}^{\mathrm{T}} & \boldsymbol{K}_{\rho \rho}
\end{array}\right]\left(\begin{array}{c}
\boldsymbol{\xi}_{A} \\
\boldsymbol{\rho}
\end{array}\right)=\left(\begin{array}{c}
\boldsymbol{f}_{A} \\
\mathbf{0}
\end{array}\right)
$$

where $\boldsymbol{\xi}_{A} \in \mathbb{R}^{6}$ denotes the nodal displacement vector at $A ; \boldsymbol{\rho} \in \mathbb{R}^{8}$ denotes the collection of the first order bending modes of three actuated limbs in conjunction with the torsional deflections of the $\mathrm{A} / \mathrm{C}$ wrist; $\boldsymbol{f}_{A} \in \mathbb{R}^{6}$ denotes the interface forces applied at $A$.

Then, we model dynamics of the tool-tool holder by treating the tool as a 2-node spatial beam element [24] while the tool holder as a rigid body such that

$$
\left[\begin{array}{cc}
\boldsymbol{M}_{\xi_{B} \xi_{B}} & \boldsymbol{M}_{\xi_{B} \xi_{C}} \\
\boldsymbol{M}_{\xi_{B} \xi_{C}}^{\mathrm{T}} & \boldsymbol{M}_{\xi_{C} \xi_{C}}
\end{array}\right]\left(\begin{array}{l}
\ddot{\boldsymbol{\xi}}_{B} \\
\ddot{\boldsymbol{\xi}}_{C}
\end{array}\right)+\left[\begin{array}{ll}
\boldsymbol{K}_{\xi_{B} \xi_{B}} & \boldsymbol{K}_{\xi_{B} \xi_{C}} \\
\boldsymbol{K}_{\xi_{B} \xi_{C}}^{\mathrm{T}} & \boldsymbol{K}_{\xi_{C} \xi_{C}}
\end{array}\right]\left(\begin{array}{l}
\boldsymbol{\xi}_{B} \\
\boldsymbol{\xi}_{C}
\end{array}\right)=\left(\begin{array}{l}
\boldsymbol{f}_{B} \\
\boldsymbol{f}_{C}
\end{array}\right)
$$




$$
\boldsymbol{\xi}_{C}=\left(\begin{array}{l}
\boldsymbol{\delta} \\
\boldsymbol{\varepsilon}
\end{array}\right), \quad \boldsymbol{\delta}=\left(\begin{array}{l}
\delta_{u} \\
\delta_{v} \\
\delta_{w}
\end{array}\right), \quad \boldsymbol{\varepsilon}=\left(\begin{array}{l}
\varepsilon_{u} \\
\varepsilon_{v} \\
\varepsilon_{w}
\end{array}\right), \quad \boldsymbol{f}_{C}=\left(\begin{array}{l}
\boldsymbol{f} \\
\mathbf{0}
\end{array}\right), \quad \boldsymbol{f}=\left(\begin{array}{l}
f_{u} \\
f_{v} \\
f_{w}
\end{array}\right)
$$

where $\xi_{B} \in \mathbb{R}^{6}$ and $\xi_{C} \in \mathbb{R}^{6}$ denote the nodal displacement vectors at $B$ and $C$, respectively, with $\boldsymbol{\delta} \in \mathbb{R}^{3}$ and $\boldsymbol{\varepsilon} \in \mathbb{R}^{3}$ being the linear and angular displacement vectors at $C$. Similarly, $\boldsymbol{f}_{B}$ and $\boldsymbol{f}_{C}$ denote the nodal force vectors applied at the corresponding nodes with $\boldsymbol{f} \in \mathbb{R}^{3}$ within $\boldsymbol{f}_{C}$ being the cutting force vector applied at TCP. In addition, $\boldsymbol{M}_{\xi_{B} \xi_{B}}$ denotes the spatial mass matrix of the tool holder about $B$.

Thirdly, we model the interface between the spindle and tool holder as a virtual joint [25] having three linear/torsional springs along/about the axes parallel to those of $\mathcal{K}_{C}$ as shown in Fig. 1. The contact stiffness coefficients of the virtual joint can be easily identified by the IRCSA method $[26,27]$ using the measured FRFs of TCP by means of impact test [28]. These considerations lead to the following compatibility conditions

$$
\boldsymbol{f}_{A}=-\boldsymbol{f}_{B}=\boldsymbol{K}_{c}\left[\begin{array}{ll}
-\mathbf{1}_{6} & \mathbf{1}_{6}
\end{array}\right]\left(\begin{array}{l}
\boldsymbol{\xi}_{A} \\
\boldsymbol{\xi}_{B}
\end{array}\right)
$$

where $\boldsymbol{K}_{c} \in \mathbb{R}^{6 \times 6}$ denotes the stiffness matrix of the virtual joint.

Finally, merging three threads given in Eqs.(1)-(3) results in the complete dynamic model of the entire system.

$$
\begin{aligned}
& {\left[\begin{array}{ll}
\boldsymbol{M}_{\xi_{C} \xi_{C}} & \boldsymbol{M}_{\xi_{C} \xi_{D}} \\
\boldsymbol{M}_{\xi_{C} \xi_{D}}^{\mathrm{T}} & \boldsymbol{M}_{\xi_{D} \xi_{D}}
\end{array}\right]\left(\begin{array}{l}
\ddot{\boldsymbol{\xi}}_{C} \\
\ddot{\boldsymbol{\xi}}_{D}
\end{array}\right)+\left[\begin{array}{ll}
\boldsymbol{K}_{\xi_{C} \xi_{C}} & \boldsymbol{K}_{\xi_{C} \xi_{D}} \\
\boldsymbol{K}_{\xi_{C} \xi_{D}}^{\mathrm{T}} & \boldsymbol{K}_{\xi_{D} \xi_{D}}
\end{array}\right]\left(\begin{array}{l}
\boldsymbol{\xi}_{C} \\
\boldsymbol{\xi}_{D}
\end{array}\right)=\left(\begin{array}{c}
\boldsymbol{f}_{C} \\
\mathbf{0}
\end{array}\right)} \\
& \boldsymbol{M}_{\xi_{C} \xi_{D}}=\left[\begin{array}{lll}
\boldsymbol{M}_{\xi_{B} \xi_{C}} & \mathbf{0} & \mathbf{0}
\end{array}\right], \quad \boldsymbol{K}_{\xi_{C} \xi_{D}}=\left[\begin{array}{lll}
\boldsymbol{K}_{\xi_{B} \xi_{C}} & \mathbf{0} & \mathbf{0}
\end{array}\right] \\
& \boldsymbol{M}_{\xi_{D} \xi_{D}}=\left[\begin{array}{ccc}
\boldsymbol{M}_{\xi_{B} \xi_{B}} & \mathbf{0} & \mathbf{0} \\
\mathbf{0} & \boldsymbol{M}_{\xi_{A} \xi_{A}} & \boldsymbol{M}_{\xi_{A} \rho} \\
\mathbf{0} & \boldsymbol{M}_{\xi_{A} \rho}^{\mathrm{T}} & \boldsymbol{M}_{\rho \rho}
\end{array}\right], \boldsymbol{K}_{\xi_{D} \xi_{D}}=\left[\begin{array}{ccc}
\boldsymbol{K}_{\xi_{B} \xi_{B}}+\boldsymbol{K}_{c} & -\boldsymbol{K}_{c} & \mathbf{0} \\
-\boldsymbol{K}_{c}^{\mathrm{T}} & \boldsymbol{K}_{\xi_{A} \xi_{A}}+\boldsymbol{K}_{c} & \boldsymbol{K}_{\xi_{A} \rho} \\
\mathbf{0} & \boldsymbol{K}_{\xi_{A} \rho}^{\mathrm{T}} & \boldsymbol{K}_{\rho \rho}
\end{array}\right], \quad \boldsymbol{\xi}_{D}=\left(\begin{array}{l}
\boldsymbol{\xi}_{B} \\
\boldsymbol{\xi}_{A} \\
\boldsymbol{\rho}
\end{array}\right)
\end{aligned}
$$

\section{Dynamic milling process modeling}

Equipped with the equations of motion of the robot developed in Section 2 at hand, we formulate robot milling process by considering the cutting force applied at TCP. The cutting force in milling process is closely related to both chip thickness and tool geometry. As shown in Fig.2, for a cylindrical milling cutter with diameter $D$ and teeth number $N$, we first establish a rotation frame $\mathcal{K}_{i}$ fixed to tooth $i(i=1, \ldots, N)$ with its $w_{i}$ axis coincident with the $w$ axis. When the tool rotates at a constant rate $\omega$ ( rev/min), the dynamic chip thickness of tooth $i$ induced by self-excited vibration can be formulated as
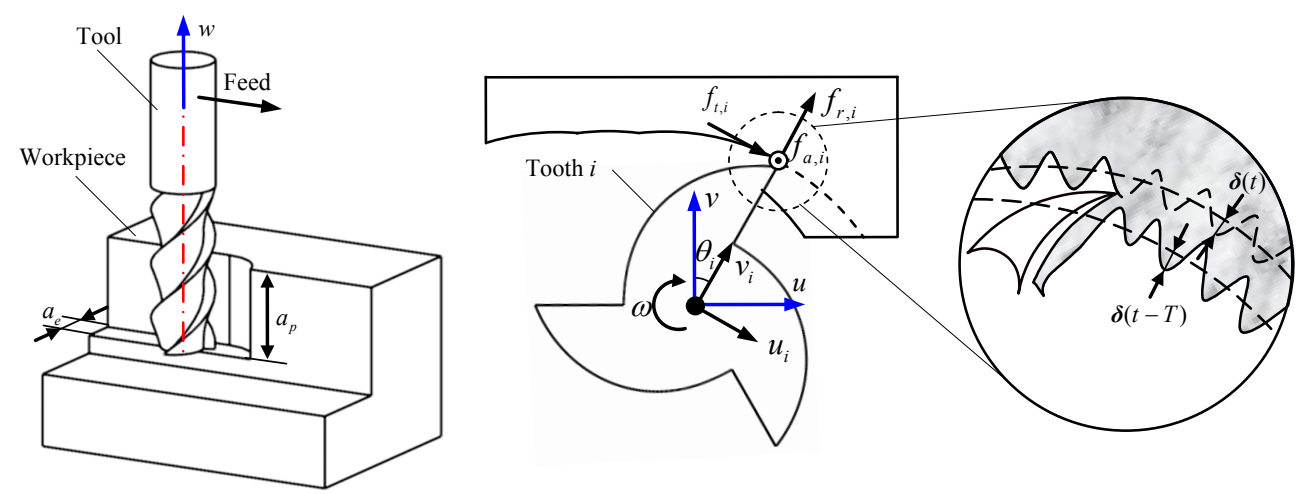

Fig.2. Dynamic model of the robot milling process 


$$
\begin{aligned}
& h_{i}(t)=\boldsymbol{v}_{i}^{\mathrm{T}}(t) \Delta \boldsymbol{\delta}(t) \\
& \boldsymbol{v}_{i}(t)=\left(\begin{array}{lll}
\sin \theta_{i}(t) & \cos \theta_{i}(t) & 0
\end{array}\right)^{\mathrm{T}}, \quad \Delta \boldsymbol{\delta}(t)=\boldsymbol{\delta}(t)-\boldsymbol{\delta}(t-T), \quad \boldsymbol{\delta}(t)=\left(\begin{array}{lll}
\delta_{u}(t) & \delta_{v}(t) & \delta_{w}(t)
\end{array}\right)^{\mathrm{T}}
\end{aligned}
$$

where $v_{i}(t)$ is the unit vector of the $v_{i}$ axis; $T=60 /(\omega N)$ is the time delay term equal to the tool passing interval; $\theta_{i}(t)$ is the radial immersion angle of tooth $i$ varying with time

$$
\theta_{i}(t)=\pi\left(\frac{\omega}{30} t+2 \frac{i-1}{N}\right)
$$

Based on the general formulations presented in Ref. [30], the dynamic cutting force applied on tooth $i$ evaluated in $\mathcal{K}_{i}$ can be expressed by

$$
\boldsymbol{f}_{i}(t)=\left(\begin{array}{l}
f_{t, i} \\
f_{r, i} \\
f_{a, i}
\end{array}\right)=a_{p} w_{i}(t)\left(\begin{array}{l}
K_{t} \\
K_{r} \\
K_{a}
\end{array}\right) h_{i}(t)
$$

where $K_{t}, K_{r}$ and $K_{a}$ denote the tangential, radial and axial cutting force coefficients; $a_{p}$ is the axial depth of cut; $w_{i}(t)$ is the unit windows function determining whether tooth $i$ is in the immersion zone

$$
w_{i}(t)=\left\{\begin{array}{cc}
1 & \theta_{\mathrm{en}} \leq \theta_{i}(t) \leq \theta_{\mathrm{ex}} \\
0 & \text { others }
\end{array}\right.
$$

where $\theta_{\mathrm{en}}$ and $\theta_{\mathrm{ex}}$ represent the entry and exit angles; $\theta_{\mathrm{en}}=0$ and $\theta_{\mathrm{ex}}=\arccos \left(1-2 a_{e} / D\right)$ for up milling, $\theta_{\mathrm{en}}=\pi-\arccos \left(1-2 a_{e} / D\right)$ and $\theta_{\mathrm{ex}}=\pi$ for down milling; $a_{e}$ is the radial depth of cut.

Transforming Eq.(7) into $\mathcal{K}_{C}$ and summing the cutting forces contributed by all teeth, the total cutting force can be formulated as

$$
\begin{gathered}
\boldsymbol{f}(t)=\sum_{i=1}^{N} \boldsymbol{R}_{i} \boldsymbol{f}_{i}(t)=a_{p} \boldsymbol{T}(t) \Delta \boldsymbol{\delta}(t) \\
\boldsymbol{R}_{i}=\left[\begin{array}{ccc}
\cos \theta_{i} & \sin \theta_{i} & 0 \\
-\sin \theta_{i} & \cos \theta_{i} & 0 \\
0 & 0 & 1
\end{array}\right], \quad \boldsymbol{T}(t)=\sum_{i=1}^{N} w_{i}(t) \boldsymbol{R}_{i}\left(\begin{array}{c}
K_{t} \\
K_{r} \\
K_{a}
\end{array}\right) \boldsymbol{v}_{i}^{\mathrm{T}}(t)
\end{gathered}
$$

where $\boldsymbol{R}_{i}$ denotes the orientation matrix of $\mathcal{K}_{i}$ with respect to $\mathcal{K}_{C} ; \boldsymbol{T}(t)$ denotes the time-periodic directional matrix of the dynamic cutting force.

Substituting Eq.(9) into Eq.(4) leads to the dynamic model of the robot milling process

$$
\left[\begin{array}{ll}
\boldsymbol{M}_{\delta \delta} & \boldsymbol{M}_{\delta \xi} \\
\boldsymbol{M}_{\delta \xi}^{\mathrm{T}} & \boldsymbol{M}_{\xi \xi}
\end{array}\right]\left(\begin{array}{l}
\ddot{\boldsymbol{\delta}}(t) \\
\ddot{\boldsymbol{\xi}}(t)
\end{array}\right)+\left[\begin{array}{ll}
\boldsymbol{K}_{\delta \delta} & \boldsymbol{K}_{\delta \xi} \\
\boldsymbol{K}_{\delta \xi}^{\mathrm{T}} & \boldsymbol{K}_{\xi \xi}
\end{array}\right]\left(\begin{array}{l}
\boldsymbol{\delta}(t) \\
\boldsymbol{\xi}(t)
\end{array}\right)=\left(\begin{array}{c}
a_{p} \boldsymbol{T}(t)(\boldsymbol{\delta}(t)-\boldsymbol{\delta}(t-T)) \\
\mathbf{0}
\end{array}\right), \quad \boldsymbol{\xi}=\left(\begin{array}{c}
\boldsymbol{\varepsilon} \\
\boldsymbol{\xi}_{D}
\end{array}\right)
$$

It can be seen from Eq. (10) that there exists not only the regenerative effect, but also structural mode coupling and load mode coupling effects in the robot milling process. Thus, the proposed model can be used to predict the stability lobes influenced by various effects.

\section{Stability analysis}

In this section, an improved stability analysis method with high efficiency is proposed at first. Then, the pose-dependent stability lobes of the TriMule robot are investigated. Finally, the distributions of limiting stable depth of cut over entire workspace, and with feed directions are predicted to guide optimizations of cutting parameters and configurations. 


\subsection{Improved full-discretization method}

The improved stability analysis method proposed here is based on the well-known full-discretization method (FDM) [18]. To investigate the influence of specific modes on stability lobe, the dynamic model of the robot milling process in Eq.(10) is first transformed into modal space. The relationship between modal coordinate $\boldsymbol{p}(t)$ and physic coordinate is

$$
\left(\begin{array}{l}
\delta(t) \\
\xi(t)
\end{array}\right)=\phi p(t), \quad \phi=\left[\begin{array}{l}
\phi \\
\phi_{2}
\end{array}\right]
$$

where $\phi$ denotes the mode shape matrix. The column vectors of matrix $\phi$ denote the specific mode shape vectors of the system considered in the stability analysis.

Substituting Eq.(11) into Eq.(10), the dynamic model expressed in modal space is derived as

$$
\begin{gathered}
\boldsymbol{M}_{p} \ddot{\boldsymbol{p}}(t)+\boldsymbol{C}_{p} \dot{\boldsymbol{p}}(t)+\boldsymbol{K}_{p} \boldsymbol{p}(t)=a_{p} \boldsymbol{\phi}^{\mathrm{T}} \boldsymbol{T}(t) \boldsymbol{\phi}[\boldsymbol{p}(t)-\boldsymbol{p}(t-T)] \\
\boldsymbol{M}_{p}=\boldsymbol{\phi}^{\mathrm{T}} \boldsymbol{M} \boldsymbol{\phi}, \quad \boldsymbol{C}_{p}=\boldsymbol{\phi}^{\mathrm{T}} \boldsymbol{C} \boldsymbol{\phi}, \quad \boldsymbol{K}_{p}=\boldsymbol{\phi}^{\mathrm{T}} \boldsymbol{K} \boldsymbol{\phi}
\end{gathered}
$$

where $C$ represents the proportional damping matrix.

Based on the state space method, Eq.(12) can be transformed into the following form

$$
\begin{gathered}
\dot{\boldsymbol{y}}(t)=\boldsymbol{A} \boldsymbol{y}(t)+\boldsymbol{B}(t)(\boldsymbol{y}(t)-\boldsymbol{y}(t-T)) \\
\boldsymbol{y}(t)=\left(\begin{array}{c}
\boldsymbol{p}(t) \\
\boldsymbol{v}(t)
\end{array}\right), \boldsymbol{v}(t)=\boldsymbol{M}_{p} \dot{\boldsymbol{p}}(t)+\boldsymbol{C}_{p} \boldsymbol{p}(t) / 2 \\
\boldsymbol{A}=\left[\begin{array}{cc}
-\boldsymbol{M}_{p}^{-1} \boldsymbol{C}_{p} / 2 & \boldsymbol{M}_{p}^{-1} \\
\boldsymbol{C}_{p} \boldsymbol{M}_{p}^{-1} \boldsymbol{C}_{p} / 4-\boldsymbol{K}_{p} & -\boldsymbol{C}_{p} \boldsymbol{M}_{p}^{-1} / 2
\end{array}\right], \boldsymbol{B}(t)=\left[\begin{array}{cc}
\mathbf{0} & \mathbf{0} \\
a_{p} \boldsymbol{\phi}^{\mathrm{T}} \boldsymbol{T}(t) \boldsymbol{\phi} & \mathbf{0}
\end{array}\right]
\end{gathered}
$$

where $\boldsymbol{A}$ denotes the constant coefficient matrix; $\boldsymbol{B}(t)$ denotes the time-periodic coefficient matrix with the principal period at tooth passing interval $T$, i.e., $\boldsymbol{B}(t+T)=\boldsymbol{B}(t)$.

To solve Eq.(13) numerically, $T$ is divided into $m-1$ time intervals (length $\tau=T /(m-1))$ firstly. Based on the direct integration scheme [18], the response function of Eq.(13) at any time $t\left(t_{k} \leq t \leq t_{k+1}, t_{k}=(k-1) \tau, k=1, \ldots, m\right)$ with initial condition $\boldsymbol{y}_{k}=\boldsymbol{y}\left(t_{k}\right)$ can be obtained as

$$
\boldsymbol{y}(t)=e^{A\left(t-t_{k}\right)} \boldsymbol{y}_{k}+\int_{t_{k}}^{t} e^{A(t-\lambda)} \boldsymbol{B}(\lambda)(\boldsymbol{y}(\lambda)-\boldsymbol{y}(\lambda-T)) d \lambda
$$

According to Eq.(14), $\boldsymbol{y}_{k+1}$ can be obtained as

$$
\boldsymbol{y}_{k+1}=e^{A \tau} \boldsymbol{y}_{k}+\int_{0}^{\tau} e^{A \lambda} \boldsymbol{B}\left(t_{k+1}-\lambda\right)\left(\boldsymbol{y}\left(t_{k+1}-\lambda\right)-\boldsymbol{y}\left(t_{k+1}-\lambda-T\right)\right) d \lambda
$$

The easiest way to handle the integral items $\boldsymbol{B}\left(t_{k+1}-\lambda\right), \boldsymbol{y}\left(t_{k+1}-\lambda\right)$ and $\boldsymbol{y}\left(t_{k+1}-\lambda-T\right)$ in Eq.(15) is to linearly approximate it using two boundary values, thus

$$
\begin{gathered}
\boldsymbol{y}\left(t_{k+1}-\lambda-T\right) \doteq \boldsymbol{y}_{k+1-m}+\lambda\left(\boldsymbol{y}_{k-m}-\boldsymbol{y}_{k+1-m}\right) / \tau \\
\boldsymbol{y}\left(t_{k+1}-\lambda\right) \doteq \boldsymbol{y}_{k+1}+\lambda\left(\boldsymbol{y}_{k}-\boldsymbol{y}_{k+1}\right) / \tau \\
\boldsymbol{B}\left(t_{k+1}-\lambda\right) \doteq \boldsymbol{B}_{k+1}+\lambda\left(\boldsymbol{B}_{k}-\boldsymbol{B}_{k+1}\right) / \tau \\
\boldsymbol{B}_{k+1}=\boldsymbol{B}\left(t_{k+1}\right), \quad \boldsymbol{B}_{k}=\boldsymbol{B}\left(t_{k}\right)
\end{gathered}
$$

Then, substituting Eqs.(16)-(18) into Eq.(15), gives

$$
\boldsymbol{y}_{k+1}=\left(\boldsymbol{P}_{0}+\boldsymbol{P}_{k}\right) \boldsymbol{y}_{k}+\boldsymbol{P}_{k+1} \boldsymbol{y}_{k+1}+\boldsymbol{P}_{k} \boldsymbol{y}_{k-m}+\boldsymbol{P}_{k+1} \boldsymbol{y}_{k+1-m}
$$

where 


$$
\begin{gathered}
\boldsymbol{P}_{0}=\boldsymbol{\Phi}_{0}, \quad \boldsymbol{P}_{k}=\left(\boldsymbol{\Phi}_{3} / \tau^{2}\right) \boldsymbol{B}_{k}+\left(\boldsymbol{\Phi}_{2} / \tau-\boldsymbol{\Phi}_{3} / \tau^{2}\right) \boldsymbol{B}_{k+1}, \quad \boldsymbol{P}_{k+1}=\left(\boldsymbol{\Phi}_{2} / \tau-\boldsymbol{\Phi}_{3} / \tau^{2}\right) \boldsymbol{B}_{k}+\left(\boldsymbol{\Phi}_{1}-2 \boldsymbol{\Phi}_{2} / \tau+\boldsymbol{\Phi}_{3} / \tau^{2}\right) \boldsymbol{B}_{k+1} \\
\boldsymbol{\Phi}_{0}=e^{A \tau}, \quad \boldsymbol{\Phi}_{1}=\int_{0}^{\tau} e^{A \lambda} d \lambda=\boldsymbol{A}^{-1}\left(\boldsymbol{\Phi}_{0}-\boldsymbol{I}\right), \quad \boldsymbol{\Phi}_{2}=\int_{0}^{\tau} \lambda e^{A \lambda} d \lambda=\boldsymbol{A}^{-1}\left(\tau \boldsymbol{\Phi}_{0}-\boldsymbol{\Phi}_{1}\right), \quad \boldsymbol{\Phi}_{3}=\int_{0}^{\tau} \lambda^{2} e^{A \lambda} d \lambda=\boldsymbol{A}^{-1}\left(\tau^{2} \boldsymbol{\Phi}_{0}-2 \boldsymbol{\Phi}_{2}\right)
\end{gathered}
$$

According to Eq.(19), a discrete map matrix can be defined as

$$
\boldsymbol{z}_{k+1}=\boldsymbol{D}_{k} \boldsymbol{z}_{k}, \quad \boldsymbol{z}_{k}=\left(\begin{array}{lllll}
\boldsymbol{y}_{k}^{\mathrm{T}} & \boldsymbol{y}_{k-1}^{\mathrm{T}} & \cdots & \boldsymbol{y}_{k+1-m}^{\mathrm{T}} & \boldsymbol{y}_{k-m}^{\mathrm{T}}
\end{array}\right)^{\mathrm{T}}
$$

where

$$
\boldsymbol{D}_{k}=\left[\begin{array}{ccccc}
\boldsymbol{Q}_{k+1}\left(\boldsymbol{P}_{0}+\boldsymbol{P}_{k}\right) & \mathbf{0} & \ldots & \boldsymbol{Q}_{k+1} \boldsymbol{P}_{k+1} & \boldsymbol{Q}_{k+1} \boldsymbol{P}_{k} \\
\boldsymbol{I} & \mathbf{0} & \ldots & \mathbf{0} & \mathbf{0} \\
\mathbf{0} & \boldsymbol{I} & \ldots & \mathbf{0} & \mathbf{0} \\
\vdots & \vdots & \ddots & \vdots & \vdots \\
\mathbf{0} & \mathbf{0} & \ldots & \boldsymbol{I} & \mathbf{0}
\end{array}\right]
$$

where $\boldsymbol{Q}_{k+1}=\left[\boldsymbol{I}-\boldsymbol{P}_{k+1}\right]^{-1}$; the Moore-Penrose generalized inverse [31] of $\left[\boldsymbol{I}-\boldsymbol{P}_{k+1}\right]$ can be used to substitute it when it is singular.

Using the discrete map matrix $\boldsymbol{D}_{k}$ defined in Eq.(20), the transition matrix $\boldsymbol{\Phi}$ of the system over one periodic time interval can be easily constructed as

$$
\boldsymbol{z}_{m}=\boldsymbol{\Phi} \boldsymbol{z}_{1}, \boldsymbol{\Phi}=\boldsymbol{D}_{m-1} \boldsymbol{D}_{m-2} \ldots \boldsymbol{D}_{1}
$$

According to Floquet theory [32], the stability of the system depends on the eigenvalues of the transition matrix $\boldsymbol{\Phi}$. Only when the moduli of all of eigenvalues are less than 1, the system was stable.

It can be seen from Eq.(21) that the construction of $\boldsymbol{\Phi}$ containing a number of high-dimensional matrices multiplication operations. The specific number of multiplications is determined by the discretization of the tooth passing interval $T$. Since the discrete map matrix $\boldsymbol{D}_{k}$ is a sparse matrix, the computational time can be reduced extensively using the sparse matrices multiplication. According to Eqs.(20) and (21), the transition matrix $\boldsymbol{\Phi}_{k+1,1}$ mapping $\boldsymbol{z}_{1}$ to $\boldsymbol{z}_{k+1}$ can be expressed as

$$
\boldsymbol{\Phi}_{k+1,1}=\boldsymbol{D}_{k} \boldsymbol{\Phi}_{k, 1}
$$

where $\boldsymbol{\Phi}_{k, 1}$ denote the transition matrix mapping $\boldsymbol{z}_{1}$ to $\boldsymbol{z}_{k}$.

As shown in Fig.3, the non-zero elements in matrix $\boldsymbol{D}_{k}$ can be divided into several partitioned matrices having the following forms

$$
\boldsymbol{S}_{k, 1}=\boldsymbol{Q}_{k+1}\left(\boldsymbol{P}_{0}+\boldsymbol{P}_{k}\right), \quad \boldsymbol{S}_{k, 2}=\left[\begin{array}{ll}
\boldsymbol{Q}_{k+1} \boldsymbol{P}_{k+1} & \boldsymbol{Q}_{k+1} \boldsymbol{P}_{k}
\end{array}\right], \quad \boldsymbol{S}_{k, 3}=\boldsymbol{I}
$$

and, matrix $\boldsymbol{\Phi}_{k, 1}$ can be divided into $\boldsymbol{T}_{k, 1}, \boldsymbol{T}_{k, 2}$ and $\boldsymbol{T}_{k, 3}$ accordingly.

For this matrices multiplication, only these partitioned matrices contribute to the result. Thus, the transition matrix $\boldsymbol{\Phi}_{k+1,1}$ can be expressed as

$$
\boldsymbol{\Phi}_{k+1,1}=\left[\begin{array}{c}
\boldsymbol{S}_{k, 1} \boldsymbol{T}_{k, 1}+\boldsymbol{S}_{k, 2} \boldsymbol{T}_{k, 2} \\
\boldsymbol{T}_{k, 3}
\end{array}\right]
$$

In the construction of $\boldsymbol{\Phi}_{k+1,1}$ considering $l$ modes of the system, the multiplication of two $2 l(m+1) \times 2 l(m+1)$ matrices is reduced to two multiplications of lower dimensional matrices by the proposed method. This treatment can dramatically simplify the computational process with none scarify of accuracy. Thus, the proposed improved FDM provides an accuracy and efficient tool for the robot milling stability analysis over the entire workspace. 

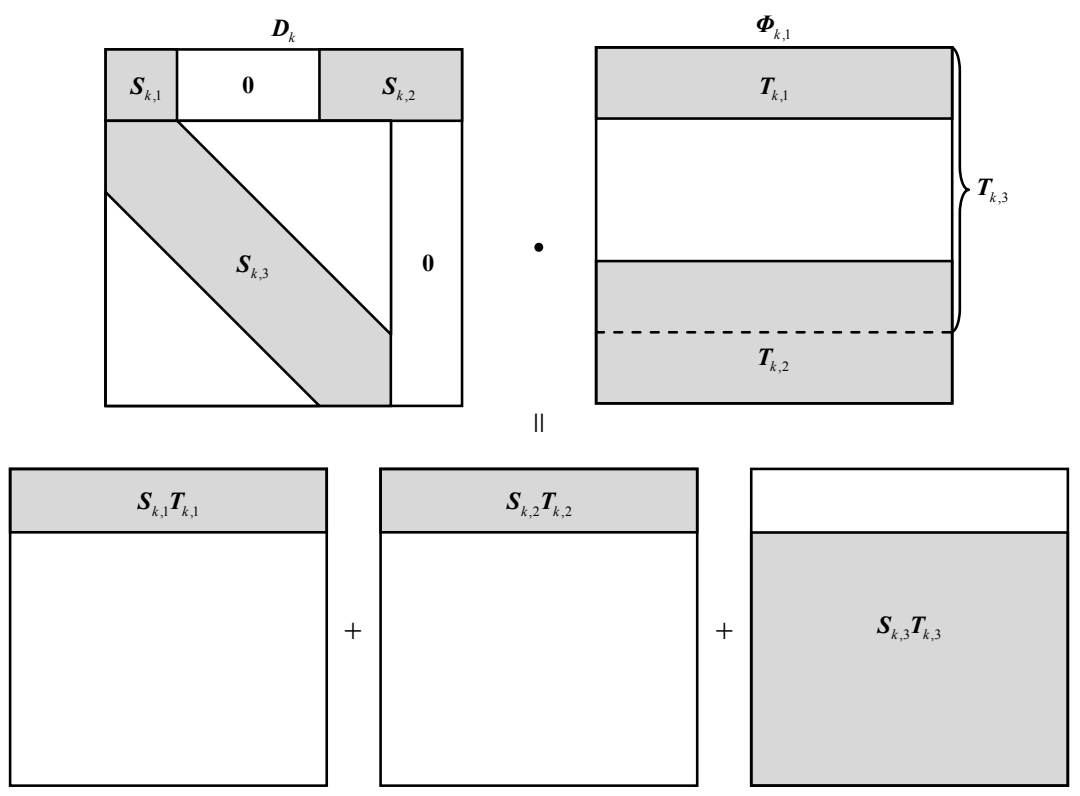

Fig.3. Multiplication operations of the partitioned matrices

\subsection{Milling stability analysis}

In this section, the pose-dependent milling stability of the TriMule robot is investigated. Fig.4 shows definitions of the workspace and some relevant structural and dimensional parameters of the robot. The reference configuration is defined as $q_{3,1}=q_{3,2}=q_{3,3}, \quad q_{3,4}=0.5\left(q_{\min }+q_{\max }\right)$. Here, $q_{3, i}(i=1 \sim 4)$ denotes the lengths of actuated and passive limbs; $q_{\max }$ and $q_{\min }$ are the maximum and minimum lengths of the RP limb. In addition, we assume that the tool axis is always perpendicular to the $x-y$ plane. As shown in Fig.4, the middle cross section of the cylinder is consequently designated as the reference plane. The dimensional and workspace parameters of the robot are given in Table 1 .
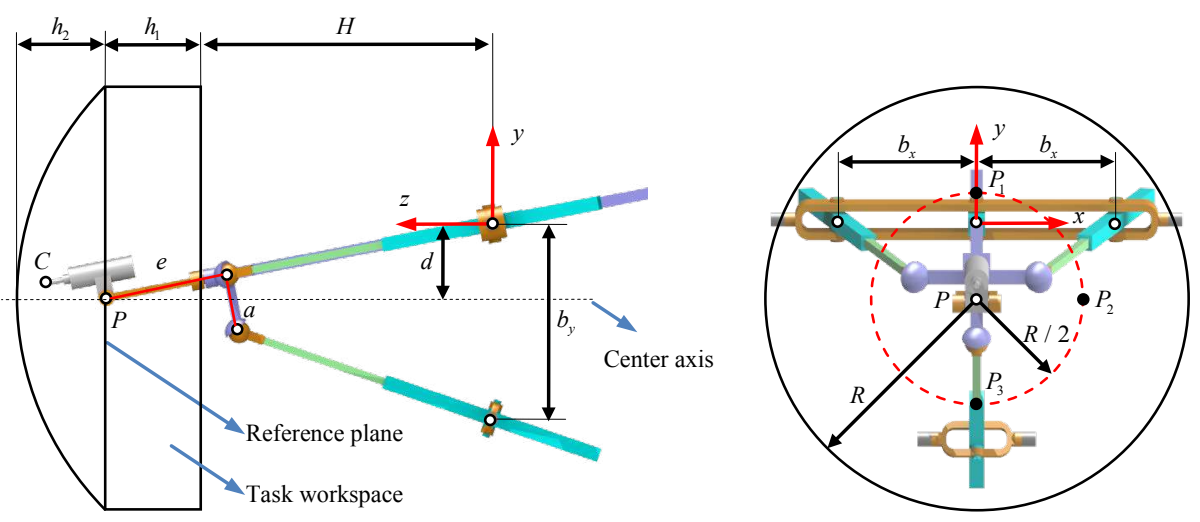

Fig.4. Task workspace of the TriMule robot

Table 1 Dimensional and workspace parameters of the TriMule robot.

\begin{tabular}{llllllll}
\hline$a(\mathrm{~m})$ & $b_{x}(\mathrm{~m})$ & $b_{y}(\mathrm{~m})$ & $d(\mathrm{~m})$ & $H(\mathrm{~m})$ & $h_{1}(\mathrm{~m})$ & $h_{2}(\mathrm{~m})$ & $R(\mathrm{~m})$ \\
\hline 0.135 & 0.320 & 0.570 & 0.190 & 1.000 & 0.240 & 0.220 & 0.600 \\
\hline
\end{tabular}

According to Ref. [7], stability lobes in low spindle speed robot milling are dominated by the lower-order structural modes of the robot. Considering that the lower-order structural modes of the robot exhibits highly pose-dependency, the

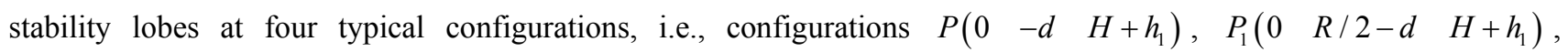


$P_{2}\left(R / 2-d \quad H+h_{1}\right)$ and $P_{3}\left(\begin{array}{lll}0 & -R / 2-d \quad H+h_{1}\end{array}\right)$ are first calculated by the proposed method. The material and cutting parameters used in the simulation are listed in Table 2. The milling tool is perpendicular to the $x-y$ plane and feeding in $x$ direction at each configuration. Fig. 5 shows the predicted stability charts. As is evident, not only the distribution of the stability boundaries, but also the limiting stable depth of cut exhibits strong pose-dependency in Titanium alloy milling process. This phenomenon is mainly resulted from the pose-dependency of lower-order structural modes of the robot, which confirms the necessity for considering the influence of cutting configurations in low spindle speed robot milling.

Table 2 Material and cutting parameters.

\begin{tabular}{cccccc}
\hline Workpiece material & $K_{t}\left(\mathrm{~N} / \mathrm{mm}^{2}\right)$ & $K_{r}\left(\mathrm{~N} / \mathrm{mm}^{2}\right)$ & $K_{a}\left(\mathrm{~N} / \mathrm{mm}^{2}\right)$ & Up/down & Radial immersion \\
\hline Titanium TC4 & 1835 & 1137 & 275 & up & $10 \%$ \\
\hline
\end{tabular}
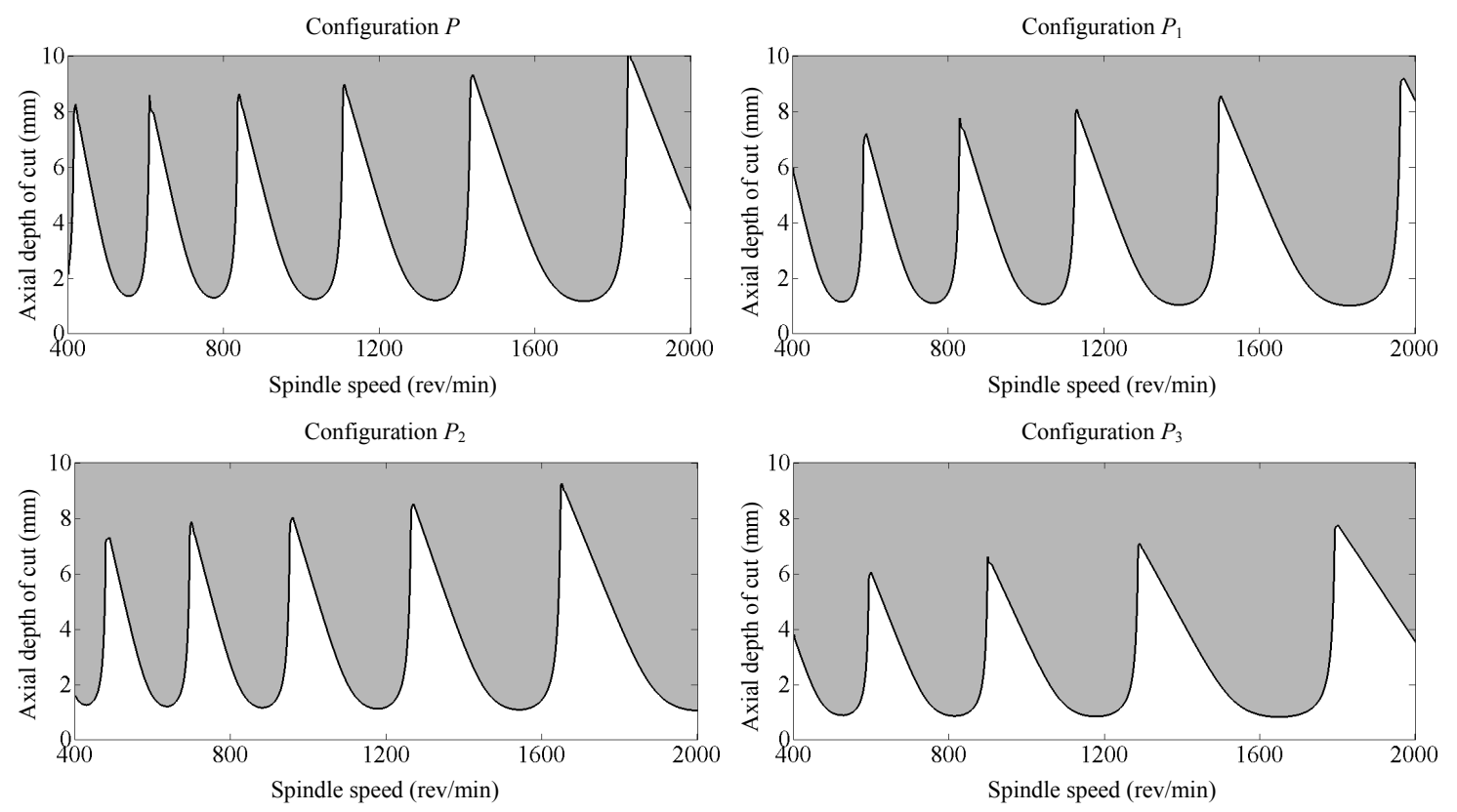

Fig.5. Predicted stability lobes for Titanium alloy at four different configurations

In order to guide the selection of cutting configuration of the robot under specific cutting condition, Fig. 6 shows the distributions of the minimum stable axial depth of cut over the entire workspace. The milling tool is perpendicular to the $x-y$ plane and feeding in $x$ direction at each configuration. It can be seen that the planar symmetrical structure of the robot

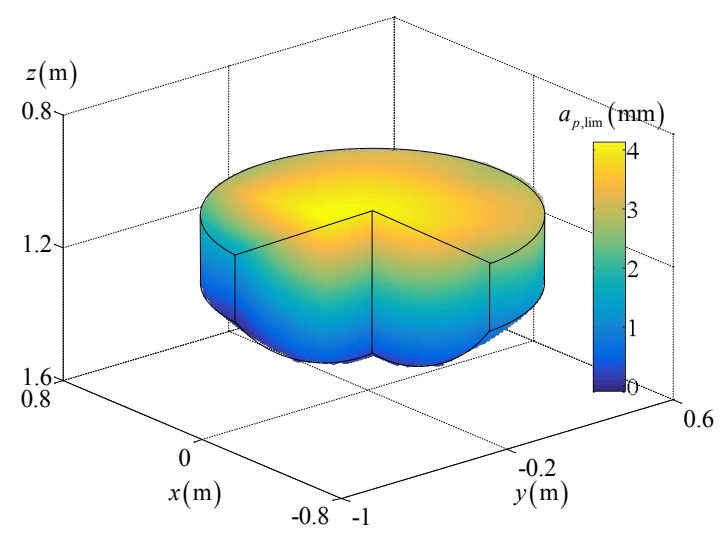

Fig.6. Distributions of the minimum stable axial depth of cut over the entire workspace 
makes the distribution of the minimum stable axial depth of cut also planar symmetry. The maximal value of them appears at the center point on the top plane of the workspace. It can also be found that these values decrease monotonically from center axis to the edge of the workspace, indicating that the TriMule robot has better cutting performance nearing the center axis of the workspace. The major features of this distribution are almost in agreement with the distributions of lower-order natural frequencies of the robot according to Ref. [26]. The minimum stable axial depth of cut is inversely proportional to the dynamic compliance of lower-order modes of the robot, indicating that the smaller dynamic compliance is, the larger stable cutting region is.

For further investigations of the influence of feed directions on milling stability, Fig.7 gives the distribution of feed-direction-dependent minimum stable axial depth of cut at the configurations along the center axis of the workspace (see Fig.4). The region inside the envelope is stable, while outside is unstable. It can be seen from Fig.7 that the larger the elongation of robot along $z$-axis is, the smaller the minimum stable axial depth of cut is. The largest and smallest minimum stable axial depths of cut appear when feeding in $x$-direction and $y$-direction at each specific configuration, respectively. This phenomenon is probably resulted from the difference of the 1st order structural mode in different major forced directions.

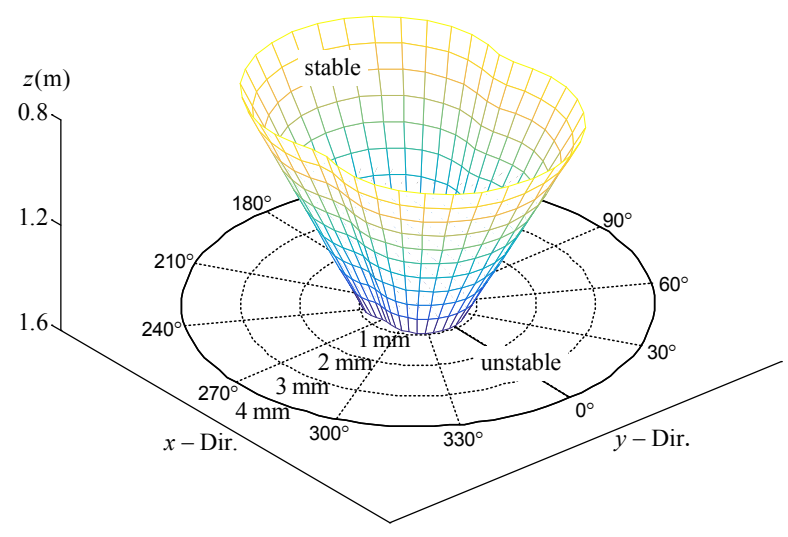

Fig.7. The distribution of the feed-direction-dependent minimum stable axial depth of cut

For a better illustration of the computational efficiency of the improved FDM, a comparison study is made on a workstation (Intel i5-3320M CPU and 8 GB RAM) as follows. Compared with 145 seconds taken by the traditional FDM at a specific configuration, only about 68 seconds were needed by the proposed improved FDM. Computational efficiency is increased by $53 \%$, leading to drastic time-savings for robot milling stability analysis over entire workspace. These observations fully consolidate the usefulness of the proposed model for predicting pose-dependent stability of the robot.

\section{Experimental validation}

This section takes a series of milling experiments with the TriMule robot (see Fig.1) to verify the efficiency of the proposed method. The contact parameters between tool holder and spindle in Eq.(3) is firstly identified through impact test at TCP. The detailed parameters are shown in Table. 3. As a check to verify the effectiveness of the proposed dynamic model, a comparison study between predicted and measured TCP FRFs was made as shown in Fig.8. It can be seen that the major features of predicted FRFs are in good agreement with the measured FRFs, and the first eighth order modes of the robot are focused on $0-300 \mathrm{~Hz}$ spectrum. The 9th-12th order modes are the local modes of spindle and tool-tool holder.

Table 3 Stiffness coefficients of contact parameters between tool holder and spindle.

\begin{tabular}{ccccccc}
\hline$k_{u}\left(10^{9} \mathrm{~N} / \mathrm{m}\right)$ & $k_{v}\left(10^{9} \mathrm{~N} / \mathrm{m}\right)$ & $k_{w}\left(10^{9} \mathrm{~N} / \mathrm{m}\right)$ & $k_{r u}\left(10^{5} \mathrm{~N} \cdot \mathrm{m} / \mathrm{rad}\right)$ & $k_{r v}\left(10^{5} \mathrm{~N} \cdot \mathrm{m} / \mathrm{rad}\right)$ & $k_{r w}\left(10^{5} \mathrm{~N} \cdot \mathrm{m} / \mathrm{rad}\right)$ \\
\hline 0.338 & 0.325 & 0.311 & 1.64 & 1.64 & 0.92 \\
\hline
\end{tabular}

$\boldsymbol{K}_{c}=\operatorname{diag}\left(k_{u}, k_{v}, k_{w}, k_{r u}, k_{r v}, k_{r w}\right)$ 

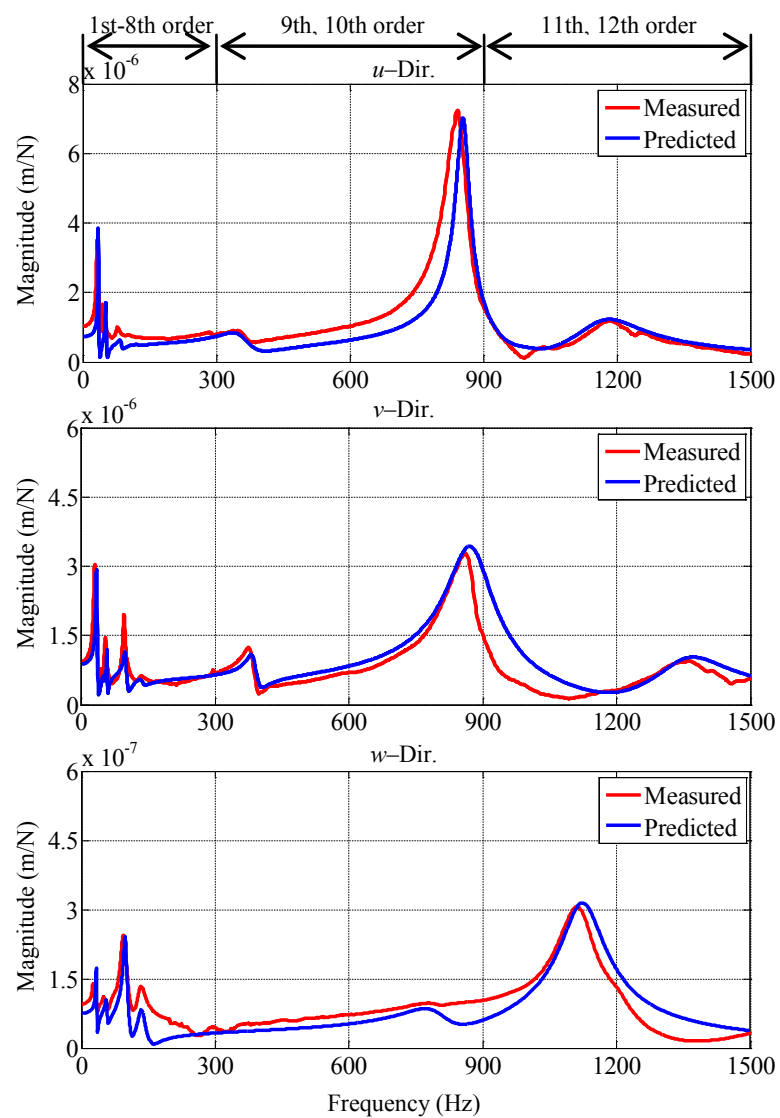

Fig.8. Comparisons between measured and predicted FRFs at TCP

In low speed milling tests, the material of the workpiece is the same as the simulations in section 4.2 , with the relevant parameters as shown in Table 2. The tool axis is aligned perpendicular to the worktable plane, and the feed direction is chosen in $x$-direction in all cutting tests. Fig.9 (a) shows the experimental setup, where a LMS dynamic analyzer is used

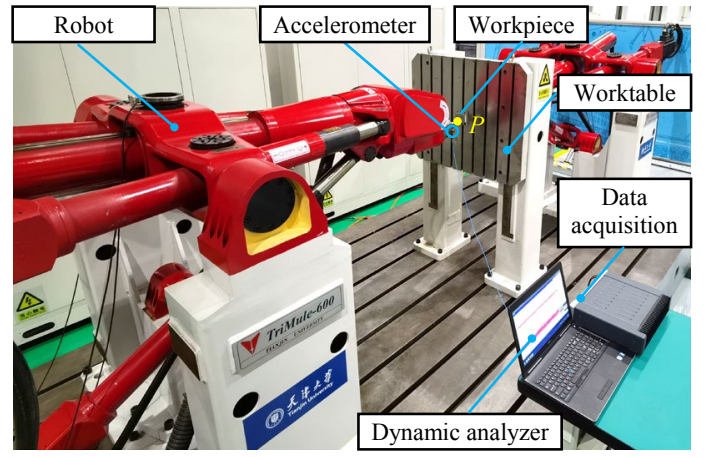

(a) Configuration $P$

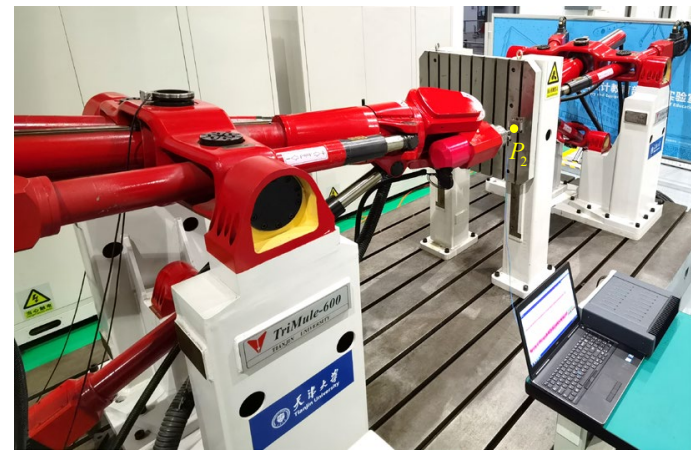

(c) Configuration $P_{2}$

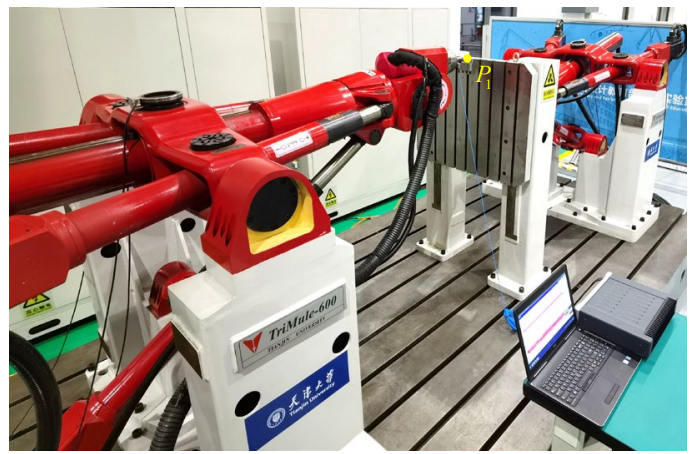

(b) Configuration $P_{1}$

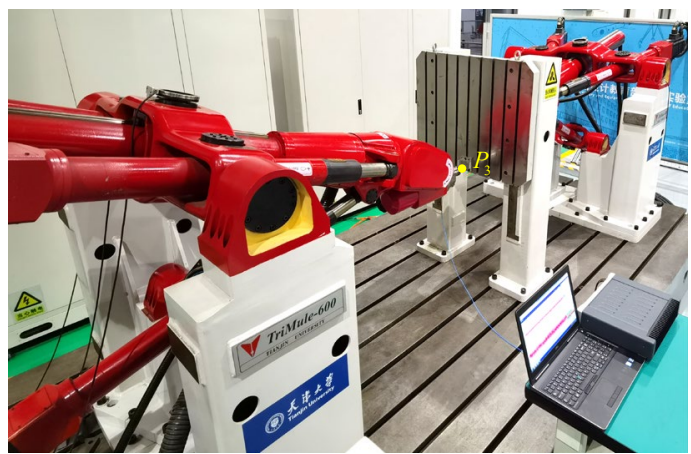

(d) Configuration $P_{3}$

Fig.9. Experimental setup and different cutting configurations of the TriMule robot in low speeding milling of Titanium alloy 
to process vibration signals measured by accelerometer (PCB 356A26). Classical 'step-cutting' strategy is applied to check the stability with the radial depth of cut keeping constant. Unstable cutting is assumed when additional dominant frequencies of vibration signals arise in the frequency spectrum.

To investigate the influence of cutting configurations on the stability, four typical configurations (as shown in Fig.9) the same as simulations were chosen. At each single configuration, 12 cutting tests with spindle speed as 800,1200 and $1600 \mathrm{rev} / \mathrm{min}$, axial depth of cut as 1, 2, 3 and $4 \mathrm{~mm}$ were conducted. The radial immersion and feed rate were assigned as $10 \%$ and $0.05 \mathrm{~mm} /$ tooth in all tests, respectively. Fig. 10 shows the comparisons of measured and predicted stability results in low spindle speed milling at four different configurations, from which it can be found that the predicted stability lobes match well with those obtained by the experiments. As is evident, though the cutting parameters are identical, the limiting axial depths of cut are different at different configurations. This phenomenon confirms that the pose-dependent lower-order structural modes of the robot lead to the pose-dependent stability.
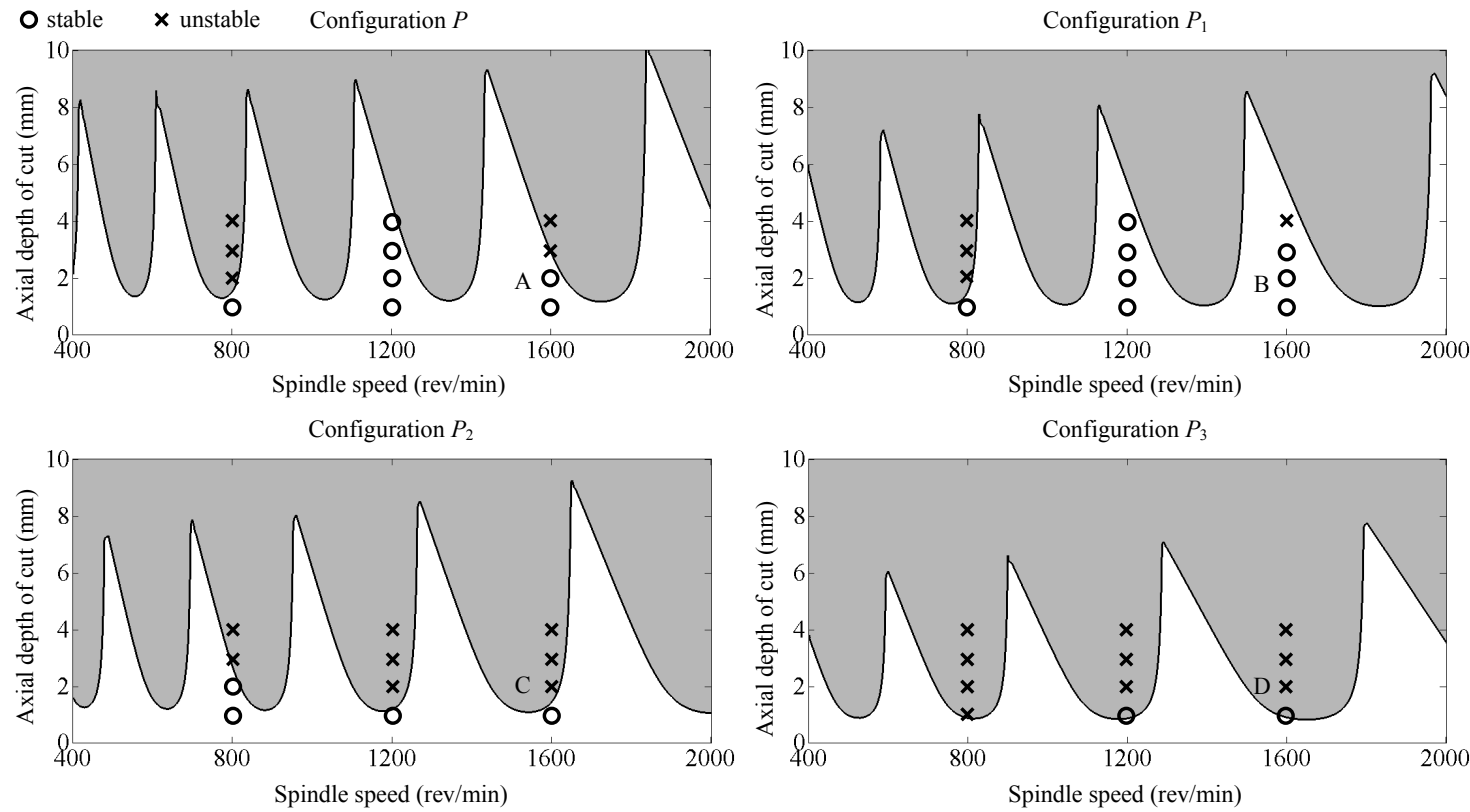

Fig.10. Comparisons of measured and predicted stability for Titanium alloy milling processes at different configurations

For a further investigation of chatter frequencies in different cutting configurations, Fig. 11 gives the finished surface characteristics and vibration signals in frequency domain of four cutting conditions (marked as A-D in Fig. 10). Obviously, conditions A-D with the same cutting parameters but different cutting configurations exhibit different stability. Undesirable finished surface is observed when chatter occurs in conditions C and D. It can be found from vibration signals that the chatter frequency are $31.09 \mathrm{~Hz}$ and $30.38 \mathrm{~Hz}$ in conditions $\mathrm{C}$ and $\mathrm{D}$ respectively, which are a little higher than the natural frequency of the 2 nd order structural mode of the robot. High frequency spindle and tool-tool holder's local modes are damped out by cutting process at low speed range cutting (400-2000 rev/min). Only tool passing frequency and its harmonics are observed in conditions $\mathrm{A}$ and $\mathrm{B}$ without chatter.

Thus, this paper provides an efficient tool for the prediction of pose-dependent chatter stability in robot milling over entire workspace, and lays a solid ground for subsequently optimizations of cutting parameters and cutting configurations.

\section{Conclusion}

This paper deals with the pose-dependent milling stability analysis of a novel 5-DOF hybrid robot named TriMule. The conclusions are drawn as follows

(1) By combining RCSA method with experimental modal analysis, a dynamic model of the TriMule robot which can predict its full frequency spectrum dynamic behaviors within the entire workspace is first established. Then, a robot dynamic milling process model considering regenerative effect, structural and load mode coupling effects is derived. By introducing sparse matrix multiplication operations in the construction of the system transition matrix, an improved full-discretization method for stability analysis is proposed. These treatments lead to dramatic time-savings in the stability analysis over entire workspace. The proposed method can be used to predict the limiting stability boundaries of the robot over entire workspace with sufficient efficiency and accuracy. 

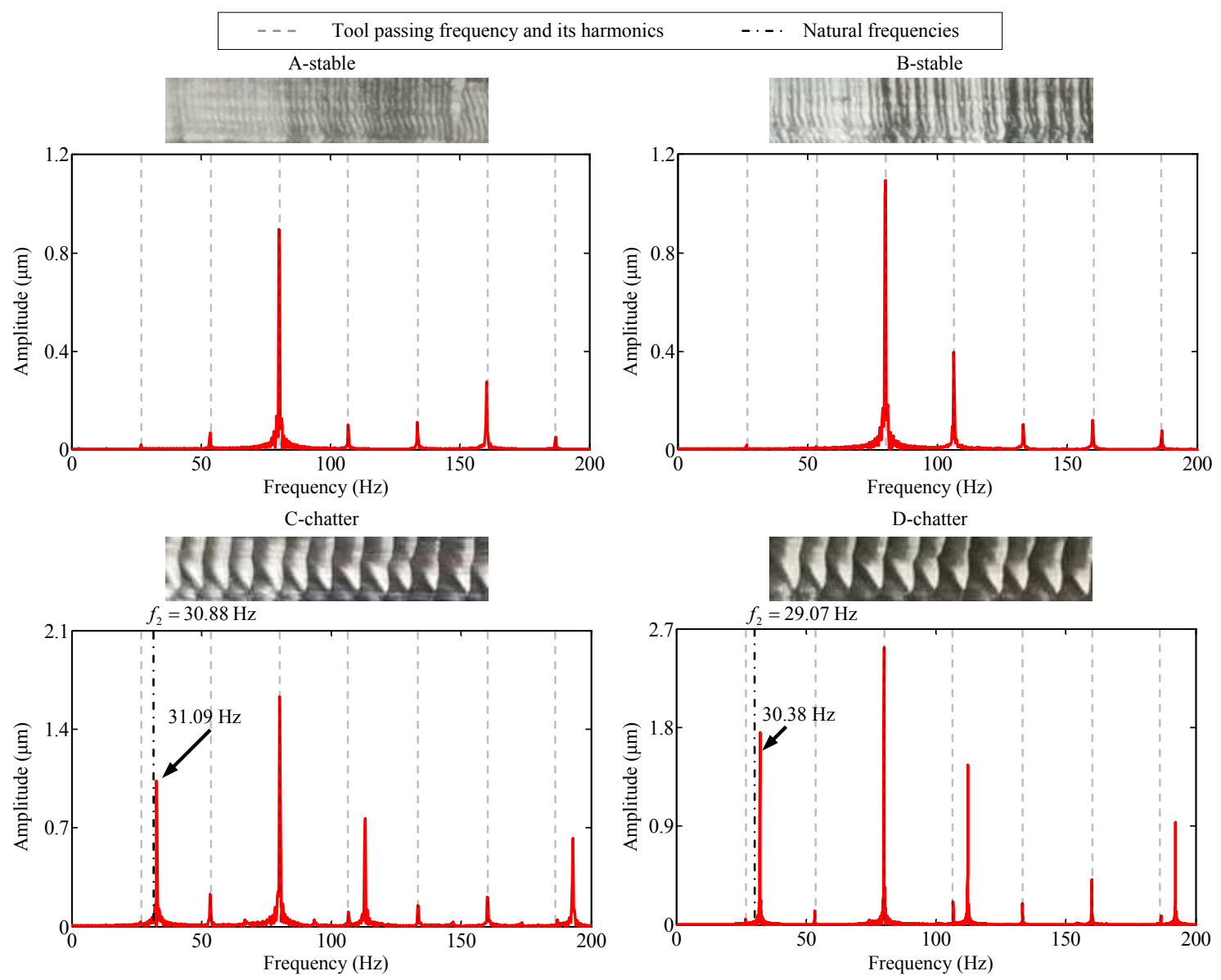

Fig.11. Comparisons of measured surface characteristic and spectrums of vibrations signals at different configurations

(2) The milling stability of the TriMule robot has been analyzed using the proposed method. Simulation results show that the stability lobes exhibit highly pose-dependency, which is mainly resulted from the pose-dependency of the lower-order structural modes of the robot. The limiting stable depth of cut decreases monotonically with the elongation of the robot along $z$-axis, and is largest in the center of top plane of the workspace. At a specific configuration, the largest limiting stable depth of cut appears when feeding in $x$-direction. The effectiveness of the proposed method has been verified by a comparison study against experimental cutting tests. The chatter frequencies appeared close to the 2 nd order structural mode of the robot in low spindle speed milling. Thus, the proposed method and analysis results in this paper lay a solid ground for further optimizations of cutting parameters and configurations in robot milling.

\section{Declarations}

Funding: This work is partially supported by National Key Research and Development Program of China (Grant No. 2019YFA0706702), National Key Research and Development Program of China (Grant No. 2019YFB1704802) and National Natural Science Foundation of China (Grant No. 52075365).

\section{Conflicts of interest/Competing interests:}

We wish to confirm that there are no known conflicts of interest associated with this publication and there has been no significant financial support for this work that could have influenced its outcome.

We confirm that the manuscript has been read and approved by all named authors and that there are no other persons who satisfied the criteria for authorship but are not listed. We further confirm that the order of authors listed in the manuscript has been approved by all of us.

We confirm that we have given due consideration to the protection of intellectual property associated with this work and that there are no impediments to publication, including the timing of publication, with respect to intellectual property. In so doing we confirm that we have followed the regulations of our institutions concerning intellectual property.

Availability of data and material: Not applicable. 


\section{Code availability: Not applicable.}

\section{Authors' contributions:}

Long $\mathrm{Wu}$ : contributed to the conception of the study, performed the data analyses and wrote the manuscript, performed the experiment;

Guofeng Wang: contributed to analysis and manuscript preparation;

Haitao Liu: performed the experiment;

Tian Huang: contributed to the conception of the study.

\section{References}

[1] M. Weck, D. Staimer, Parallel kinematic machine tools-current state and future potentials, CIRP Ann. Manuf. Technol. 51(2) (2002) 671-683.

[2] H.E. Merritt, Theory of self-excited machine-tool chatter, Trans. ASME J. Eng. Ind. 87 (11) (1965) 447-454.

[3] L. Wu, G. Wang, H. Liu, T. Huang, An approach for elastodynamic modeling of hybrid robots based on substructure synthesis technique, Mech. Mach. Theory 123 (2018) 124-136.

[4] C. Dong, H. Liu, T. Huang, D. G. Chetwynd, A Screw Theory-Based Semi-Analytical Approach for Elastodynamics of the Tricept Robot, ASME J. Mech. Robot. 11(3) (2019) 031005.

[5] J. Tlusty, F. Ismail, Basic non-linearity in machining chatter, CIRP Ann. Manuf. Technol. 30 (1) (1981) $299-304$.

[6] Z. Pan, H. Zhang, Z. Zhu, J. Wang, Chatter analysis of robotic machining process, J. Mater. Process. Technol. 173 (3) (2006) 301-309.

[7] M. Cordes, W. Hintze, Y. Altintas, Chatter stability in robotic milling, Robot. Comput. Integr. Manuf. 55 (2019) 11-18. doi:10.1016/j.rcim.2018.07.004.

[8] S. Mejri, V. Gagnol, T.-P. Le, L. Sabourin, P. Ray, P. Paultre, Dynamic characterization of machining robot and stability analysis, Int. J. Adv. Manuf.Technol. 82 (1-4) (2016) 351-359.

[9] L.T. Tunc, J. Shaw, Investigation of the effects of Stewart platform-type industrial robot on stability of robotic milling, Int. J. Adv. Manuf. Technol. 87 (2016) 189-199. doi:10.1007/s00170-016-8420-z.

[10] S. Mousavi, V. Gagnol, B.C. Bouzgarrou, P. Ray, Dynamic modeling and stability prediction in robotic machining, Int. J. Adv. Manuf. Technol. 88 (2017) 3053-3065. doi:10.1007/s00170-016-8938-0.

[11] M. Law, S. Ihlenfeldt, M. Wabner, Y. Altintas, R. Neugebauer, Position-dependent dynamics and stability of serial-parallel kinematic machines, CIRP Ann. Manuf. Technol. 62 (1) (2013) 375-378.

[12] Y. Altintas, E. Budak, Analytical prediction of stability lobes in milling, CIRP Ann. Manuf. Technol. 44 (1) (1995) 357-362.

[13] E. Budak, Y. Altintas, Analytical prediction of chatter stability in milling — part i: general formulation, J. Dyn. Syst. Meas. Control 120 (1) (1998) $22-30$.

[14] Sims ND, Mann B, Huyanan S (2008) Analytical prediction of chatter stability for variable pitch and variable helix milling tools. J Sound Vib 317(3-5):664-686

[15] T. Insperger, G. Stepan, Semi-discretization method for delayed systems,, International Journal for Numerical Methods in Engineering 55 (5) (2002) 503-518.

[16] T. Insperger, G. Stepan, Updated semi-discretization method for periodic delay-differential equations with discrete delay,, International Journal for Numerical Methods in Engineering 61 (1) (2004) 117-141.

[17] Long XH, Balachandran B, Mann BP (2007) Dynamics of milling processes with variable time delays. Nonlinear Dyn 47(1-3):49-63.

[18] Ding Y, Zhu LM, Zhang XJ, Ding H. A full-discretization method for prediction of milling stability. Int J Mach Tool Manuf 2010, 50(5):502-9.

[19] Ding Y, Zhu LM, Zhang XJ, Ding H. Second-order full-discretization method for milling stability prediction. Int JMach Tool Manuf 2010, 50(10):926-32.

[20] Ding Y, Niu JB, Zhu LM, Ding H (2015) Numerical integration method for stability analysis of milling with variable spindle speeds. ASME J Vib Acoust 138(1):011010-011011

[21] T. Huang, C. Dong, H. Liu, X. Qin, J. Mei, Q. Liu, M. Wang, Five-degree-of-freedom parallel robot with multi-shaft rotary brackets, Pub. No. WO/2017/005015 A1, 2017

[22] T. Huang, C. Dong, H. Liu, T. Sun, D. G. Chetwynd, A simple and visually orientated approach for type synthesis of overconstrained 1T2R parallel mechanisms, Robotica (2018) 1-13.

[23] L. Wu, C. Dong, G. Wang, H. Liu, T. Huang, An approach to predict lower-order dynamic behaviors of a 5-DOF hybrid robot using a minimum set of generalized coordinates, Robot. Comput. Integr. Manuf. 67 (2021) 102024. doi:10.1016/j.rcim.2020.102024.

[24] David H., 2004, Fundamentals of Finite Element Analysis, McGraw-Hill, New York.

[25] H. Liu, T. Huang, D. G. Chetwynd, et al., Stiffness modeling of parallel mechanisms at limb and joint/link levels, IEEE Trans. Rob. 33 (3) (2017) 734-741.

[26] T.L. Schmitz, R.R. Donalson, Predicting High-Speed Machining Dynamics by Substructure Analysis, CIRP Annals 49 (1) (2000) $303-308$.

[27] M. Namazi, Y. Altintas, T. Abe, N. Rajapakse, Modeling and identification of tool holder-spindle interface dynamics, Int. J. Mach. Tools Manuf. 47 (2007) 1333-1341. doi:10.1016/j.ijmachtools.2006.08.003.

[28] D. J. Ewins, Modal testing: theory, practice, and application, Research Sstudies Press, Baldok, Hertfordshire, UK, 2000.

[29] S. Tayeb, D. Givoli, Optimal modal reduction of dynamic subsystems: Extensions and improvements, Int. J. Numer. Methods Eng. 85 (2011) $1-30$.

[30] Y. Altintas, Manufacturing Automation: Metal Cutting Mechanics, Machine Tool Vibrations, and CNC Design, 2 edition, Cambridge University Press, Cambridge, New York, 2012.

[31] C.D. Meyer, in: Matrix Analysis and Applied Linear Algebra, SIAM, Philadelphia, 2000. 
[32] M. Farkas, in: Periodic Motions, Springer-Verlag, New York, 1994. 